\title{
A Visualização de Informação e a Transparência de Dados Públicos
}

\author{
Melise M. V. de Paula ${ }^{1}$, Fernanda C. Ribeiro ${ }^{1}$, Miriam Chaves ${ }^{2}$, Sergio A. \\ Rodrigues $^{3}$, Jano M. de Souza ${ }^{3}$ \\ ${ }^{1}$ Instituto de Ciências Exatas - Universidade Federal de Itajubá (UNIFEI) \\ Av. BPS, 1303 - Bairro Pinheirinho - 37500-903 - Itajubá - MG - Brasil \\ ${ }^{2}$ Ministério do Planejamento, Orçamento e Gestão \\ Esplanada dos Ministérios - Brasília - DF - Brasil \\ ${ }^{3}$ Programa de Engenharia de Sistemas e Computação - COPPE/UFRJ \\ Caixa Postal: 68511 - 21941-972 - Rio de Janeiro - RJ - Brasil \\ \{melisepaula, fernandafcrk, miriambfc\}@gmail.com, \{sergio, \\ jano\} @cos.ufrj.br
}

\begin{abstract}
The current global scenario holds an information overload that is available to support people during their decision making process. Several areas, including Government, have to deal with information overconsumption. As a result, this situation may jeopardize communication between Govern and Society and their decisions. The Information Visualization (InfoVis) is a subject that develops techniques for solving information overload problems. There are several methods of InfoVis and this article aims at analyze (through experiments) the applicability of these techniques in the government.

Resumo. O contexto atual é marcado pelo excesso de informação disponível aos usuários para apoiar a tomada de decisão. Esse excesso está presente em todas as áreas, inclusive no governo. Como consequência, a comunicação com a sociedade e a tomada de decisão nos setores públicos são prejudicadas. A Visualização de Informação (InfoVis) é uma área de estudo cujo objetivo é desenvolver técnicas para solucionar este problema. $O$ objetivo deste artigo é analisar a aplicabilidade destes métodos nos setores públicos.
\end{abstract}

\section{Introdução}

O cenário atual é marcado pelo aumento na quantidade de informações disponibilizadas aos usuários para apoiar a tomada de decisões. Um dos grandes desafios do uso da tecnologia de informação é: como lidar com o excesso de informação?

A Visualização de Informações (InfoVis) é uma disciplina de estudo que vem se destacando neste cenário. O objetivo da pesquisa nesta área é auxiliar o usuário na análise e compreensão das informações (Card e Mackinlay, 1997). As técnicas desenvolvidas são utilizadas para solucionar o problema do excesso de informações e nasceram da necessidade humana de criar representações de sistemas complexos de informação (Ribeiro, 2009).

O excesso de informação está presente em todas as áreas, inclusive no governo. Este fato prejudica tanto a comunicação com a sociedade quanto a tomada de decisão 
nos setores públicos. Neste contexto, a tecnologia, mais precisamente, a InfoVis, pode ser considerada um diferencial tanto para alavancar a transparência dos dados governamentais quanto no suporte ao processo de decisão. São diversos os exemplos de governos que usam a InfoVis para publicação dos seus dados.

Por exemplo, no site do governo de Canárias (Canárias, 2011), algumas informações podem ser consultadas a partir do mapa da comunidade. Já o governo dos Estados Unidos disponibiliza uma quantidade considerável de informações através do portal Free Government Information (FGI, 2011). E no Brasil, o site do COI-PR (Comitê de Organização de Informações da Presidência da República) organiza e disponibiliza ao Presidente, aos órgãos do Governo Federal e à sociedade, informações das principais ações governamentais na forma de tabela e gráficos.

O objetivo desse artigo é apresentar um estudo que vem sendo realizado com objetivo de investigar as técnicas de InfoVis e sua aplicação no contexto governamental. A questão abordada é: quais técnicas são mais apropriadas para apoiar a tomada de decisão nos setores públicos e a comunicação com a sociedade? Neste artigo, o foco foi a divulgação de dados públicos. Em uma primeira fase do trabalho, foi realizado um experimento inicial, denominado Ensaio Piloto. O objetivo desta fase foi especificar quais técnicas de visualização seriam consideradas em uma fase posterior do trabalho.

Em seguida, foi realizado o experimento principal. Nesta fase, as técnicas selecionadas foram utilizadas juntamente com dados publicados pelo COI-PR. Os dados foram apresentados a um grupo de usuários que foram observados pelos pesquisadores envolvidos sendo possível coletar algumas informações. As visualizações utilizadas foram geradas a partir do Many Eyes, um projeto da IBM, criado por Fernanda Viégas e Martin Wattenberg, que disponibiliza aos usuários ferramentas para a criação de visualizações interativas, sendo permitido compartilhá-las com outros usuários. (Projeto Many Eyes, 2011). Vale ressaltar que, em um primeiro momento, não faz parte do escopo deste trabalho desenvolver novas técnicas. Além disso, a avaliação quanto à usabilidade das ferramentas disponibilizadas no contexto do projeto Many Eyes também não faz parte do objetivo do trabalho.

O artigo está dividido em 5 seções. Após a introdução, é apresentada a revisão da literatura considerando alguns trabalhos relacionados à InfoVis. Na terceira seção, será apresentado o Ensaio Piloto e os primeiros resultados obtidos nesta investigação. $\mathrm{Na}$ quarta seção, será detalhado o experimento principal e os resultados obtidos. Finalmente, a quinta seção apresenta as considerações finais e um direcionamento das próximas etapas deste estudo.

\section{Revisão da Literatura}

A InfoVis transforma um conjunto de dados abstratos em uma representação gráfica e interativa de forma a facilitar o seu entendimento e/ou ajudar na descoberta de novas informações (Silva, 2007; Nascimento e Ferreira, 2005). Logo, a visualização é o caminho natural para facilitar a interpretação de dados processados pelo computador.

O processo computacional que converte a informação em uma visualização é conhecido como modelo de referência para visualização. Esse modelo foi proposto por Card et al. (1999) e permite a interação do usuário com as representações visuais.

O modelo de referência possui três transformações: 
- Transformações do Dado: trabalha com o dado bruto e o armazena em uma tabela. Dado bruto é todo dado coletado de uma situação em estudo. O conjunto de dados brutos coletados pode ser heterogêneo contendo datas, medidas, imagens, vídeos, endereços de sites da Internet, entre outros.

- Mapeamento Visual: diz respeito à escolha da representação visual (linear, mapa, árvore, etc.) para o mapeamento dos dados armazenados na tabela e seu objetivo é ampliar a cognição humana.

- Transformações Visuais: consiste nas possíveis interações que o usuário realiza sobre a estrutura da representação obtendo diferentes visões. Com isso, o usuário modifica dinamicamente qualquer fatia do conjunto de dados a ser exibido, reduzindo o excesso de dados e obtendo uma informação mais precisa e clara.

É na fase do mapeamento visual que são criadas as técnicas de visualização. $\mathrm{Na}$ literatura, é possível encontrar várias classificações destas técnicas. Lengler e Eppler (2007) organizaram os métodos de visualização em uma "tabela periódica". Onde cada "elemento" é um método. Para Souza (2008), a tabela periódica é uma abordagem de como organizar, onde aplicar e como interagir com o grande número de visualizações existentes. As técnicas são subdivididas em seis categorias (Souza, 2008):

- Visualização de Dados - são as representações visuais de dados quantitativos, que podem ser encontrados nos gráficos de linha, pizza, barra ou áreas.

- Visualização de Informações - trata-se das representações visuais que transformam dados em imagens, por exemplo, os diagramas de fluxo, de entidaderelacionamento e as linhas do tempo. Esse método é capaz de ampliar a cognição (conhecimento, percepção) do usuário.

- Visualização Conceitual - representam os conceitos qualitativos, as ideias, os planos e as análises. Os mapas mentais e conceituais e os gráficos de camadas são os exemplos desse método, que são representados por círculos ou caixas conectados a outros elementos formando um relacionamento.

- Visualização Metafórica - utilizam metáforas visuais para representar um conjunto de informações complexas de modo organizado e estruturado.

- Visualização Estratégica - tratam-se das representações visuais utilizadas para a análise, comunicação e desenvolvimento de estratégias em uma organização. Por exemplo: diagrama de ciclo de vida ou de cadeia de valor.

- Visualização Composta - são as representações híbridas, ou seja, contem um ou mais métodos identificados nas categorias anteriores.

Além disso, os métodos de visualização são classificados em cinco dimensões:

- Complexidade da visualização: refere-se ao número de interdependência entre os elementos a serem visualizados. A complexidade pode ser alta ou baixa.

- Área de aplicação: visualização de dados, informação, conceito, metáfora, estratégia e conhecimento composto.

- $\quad$ Ponto de visão: subdividido em detalhe (destaca os itens individuais), visão geral (apresenta o todo) e detalhe + visão geral (ambos ao mesmo tempo).

- Tipo de ajuda esperada: convergente (reduz a complexidade através de análise e síntese) e divergente (adiciona complexidade com o objetivo de obter insight).

- Tipo de informação representada: estruturada (por exemplo: redes e hierarquias) e processo (com fases em tempos cíclicos ou contínuos seqüenciais). 
O período (linhas) representa a complexidade da visualização: quanto mais à direita uma visualização, maior é sua complexidade. O grupo (colunas) representa a área de dimensão. A cor das letras de cada elemento ilustra o tipo de informação representada: cor preta para a informação estruturada e azul para a informação de processo. Acima da sigla da visualização está o símbolo que representa o ponto de visão e o tipo de ajuda esperada. O detalhe é representado por $\alpha$, a visão geral por e a visão geral com detalhe por $\&$. O símbolo $<>$ representa o tipo de ajuda convergente e $><$ representa o divergente. Na versão interativa (Visual Literacy, 2010) da tabela periódica, é possível ver um exemplo de cada técnica, ao posicionar o mouse sobre cada "elemento".

Além das técnicas apresentadas na tabela, há outros métodos de visualização, incluindo as técnicas utilizadas para dados textuais. A seguir, são listadas três técnicas de visualização de textos, descritas pelos autores do projeto Many Eyes (Many Eyes, 2011):

- Árvores de palavras (Word Tree): permitem visualizar textos não estruturados, como livros, artigos, discursos ou poemas. O usuário pode escolher uma palavra ou uma frase e o método deve retornar os diferentes contextos em que a palavra ou frase aparece. Os contextos são dispostos em uma árvore exibindo os temas recorrentes e frases. Para exemplificar esse método foi gerado, no site do Many Eyes (Many Eyes, 2011), um Word Tree (Word Tree, 2011) com relatórios (Relatórios PAC, 2011) do PAC (Programa de Aceleração do Crescimento) disponíveis à população (Figura 1).

- Núvens de Tag (Tag Clouds): neste método, é possível identificar a freqüência das palavras em um texto. A Figura 2 mostra a técnica Tag Cloud (Tag Cloud, 2011), gerada no site Many Eyes (Many Eyes, 2011), com relatórios do PAC (Relatórios PAC, 2011).

- Rede de frases (Phrase Net): a partir desta visualização, é possível identificar a relação entre palavras em um texto.

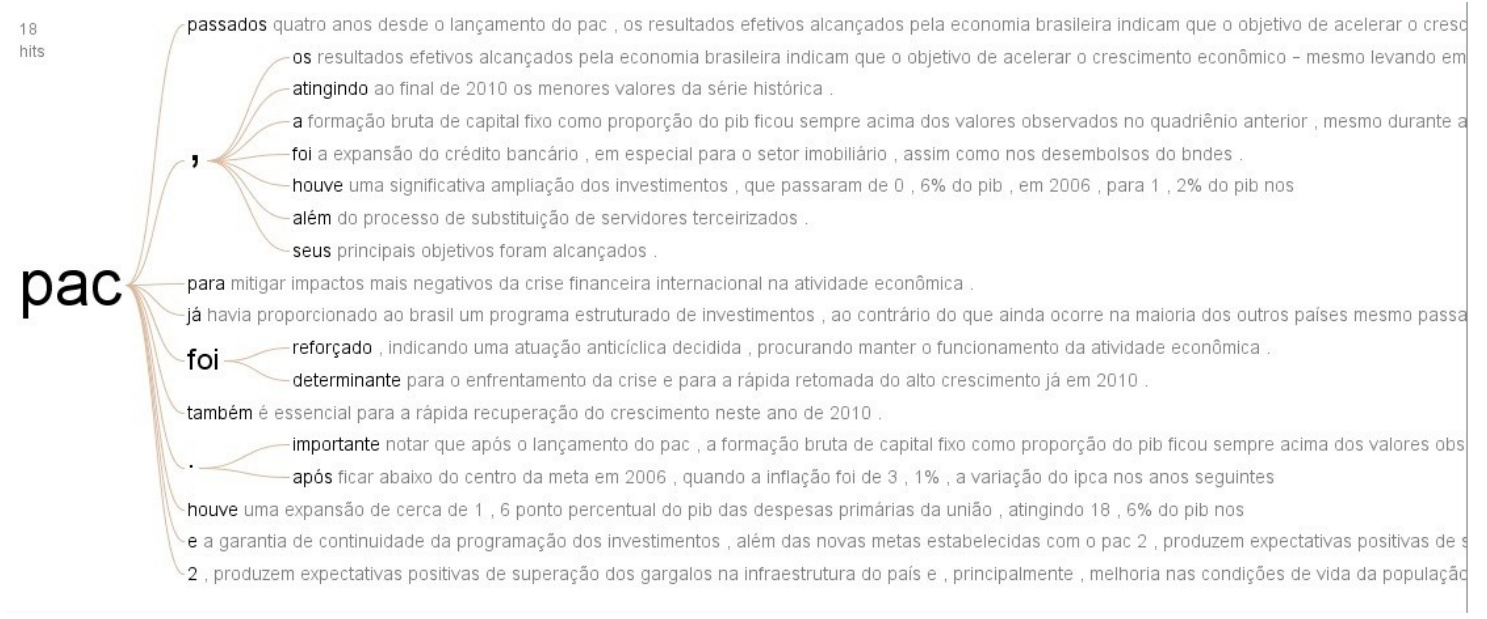

Figura 1. Word Tree com relatórios do PAC 


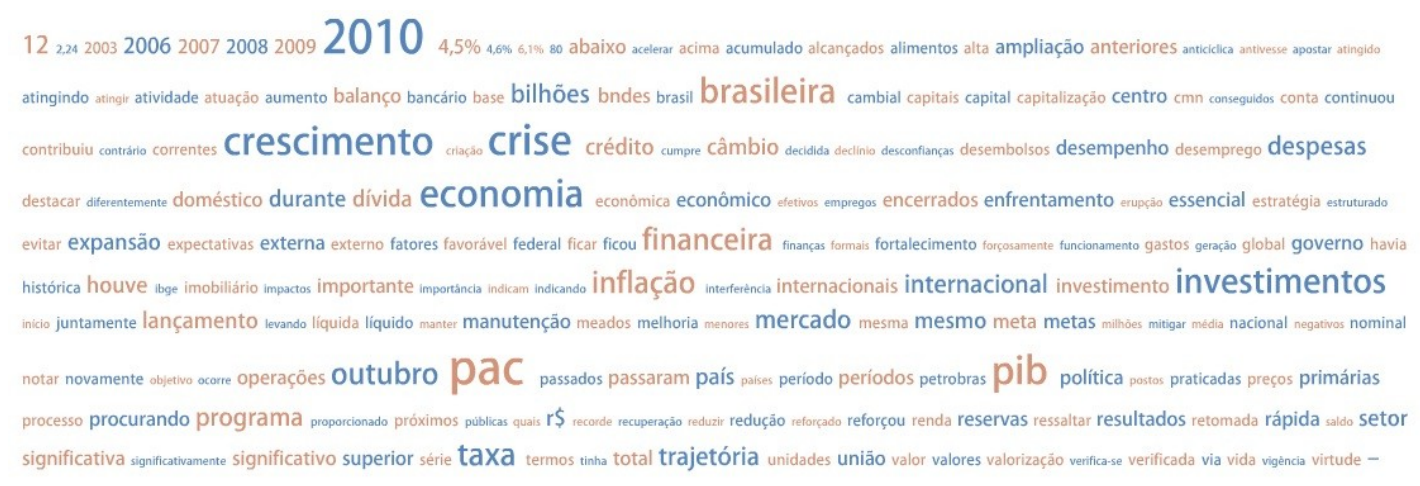

Figura 2. Tag Cloud com relatórios do PAC

\section{Ensaio Piloto}

O objetivo desta fase do trabalho foi aprimorar os procedimentos de testes e verificar sua adequação prática. Além disso, os testes realizados no ensaio piloto permitiram a seleção das técnicas de visualização a serem utilizadas em uma fase posterior do estudo.

Em um primeiro momento, foram geradas, no Many Eyes, visualizações considerando dois conjuntos de dados associados ao PAC publicados pelo COI-PR (COI, 2011):

- Número de pessoas atendidas pelo programa Luz Para Todos nos anos de 2008 e 2009.

- Quantidade de moradias do programa Minha Casa Minha Vida nos anos de 2009 e 2010.

Foram utilizadas cinco técnicas de visualização de informações: gráfico de bolhas (Bubble Chart, 2011), Brazil Map (Brazil Map, 2011), gráfico de colunas, de pizza e Treemap (Shneiderman, 1992). Para cada conjunto de dados, foram geradas as cinco visualizações. Tanto os conjuntos de dados, quanto as representações visuais estão disponíveis no site Many Eyes (Visualizações Many Eyes, 2011).

Participaram do ensaio piloto, oito pessoas com idade entre 17 e 26 anos: dois estudantes de ensino médio, cinco estudantes universitários e um professor universitário. Todos participantes declararam não ter conhecimento sobre InfoVis. Deste modo, inicialmente, foi dada uma breve explanação sobre InfoVis e o objetivo do ensaio piloto. Para cada visualização gerada, os participantes tiveram 30 segundos para analisar as informações e depois escreveram em uma folha de papel suas observações quanto às técnicas (aspectos positivos e negativos). O gráfico da Figura 3 representa a quantidade de observações positivas e negativas descritas para cada técnica. 


\section{Número de observações positivas e negativas}

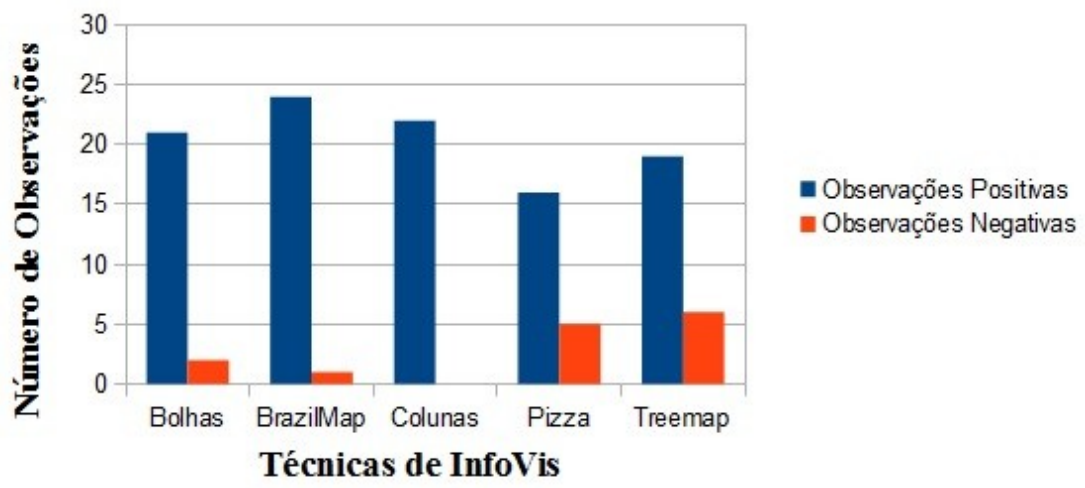

Figura 3. Número de observações positivas e negativas - Ensaio Piloto

Com base nas observações realizadas durante o ensaio piloto, foi definido o procedimento a ser utilizado no experimento principal incluindo os dados e técnicas a serem utilizados. O resultado desta fase mostrou que as técnicas BrazilMap e Gráfico de Colunas obtiveram o maior número de observações positivas e o menor número de observações negativas (Figura 3). Portanto, essas foram as técnicas escolhidas para a realização do experimento principal apresentado na seção seguinte.

\section{Experimento}

O objetivo do experimento foi comparar a aplicabilidade das técnicas BrazilMap e Gráfico de Colunas na comunicação com a sociedade. A metodologia utilizada para o experimento (e também para o ensaio piloto) foi baseada na técnica Ensaio de Interação.

Segundo Ramos (2004), essa técnica simula uma situação real de trabalho, em campo ou em laboratório, do qual participam usuários representativos da população alvo do sistema. O propósito do ensaio de interação é revelar problemas ligados à utilização real do sistema e obter dados objetivos sobre a produtividade na interação (Ramos, 2004). Contudo, essa técnica foi adaptada para se adequar as características do contexto deste trabalho.

Assim como no ensaio piloto, as visualizações foram criadas no site Many Eyes (Visualizações Many Eyes, 2011) e os conjuntos de dados (Dados Many Eyes, 2011) estão associados aos programas Bolsa Alimentação, Bolsa Escola e Bolsa Família do Governo Federal. Os dados foram retirados do site do COI-PR (COI, 2011).

Nesta fase, participaram cinco pessoas, com idade entre 19 e 47 anos: três estudantes universitários, uma recepcionista com segundo grau completo e um desempregado com ensino superior em Tecnologia em Processamento de Dados. No início do experimento, foi perguntado aos participantes se eles tinham conhecimento sobre a área de InfoVis e todos responderam que não.

O experimento foi dividido em três etapas: introdução, etapa 1 e etapa 2. Os participantes foram monitorados via screenCam (ScreenCam, 2011) com a finalidade de registrar o tempo e a interação com cada técnica em cada etapa. No ensaio piloto, os participantes tiveram 30 segundos para analisar cada técnica e todos afirmaram que o tempo não foi suficiente para interpretá-las. Desta forma, no experimento principal, os 
participantes puderam interagir com as visualizações sem uma limitação de tempo. Contudo, a duração de cada interação foi registrada.

Durante todo o experimento, os participantes utilizaram uma folha de papel e caneta para responder as perguntas e anotar suas observações. $\mathrm{O}$ experimento foi conduzido por um pesquisador que também registrava as considerações importantes a respeito da interação do participante com cada técnica.

$\mathrm{Na}$ introdução, foi apresentado um exemplo das técnicas BrasilMap e Gráfico de Colunas e foi feita a seguinte pergunta: para analisar dados relacionados aos estados brasileiros, qual técnica (BrazilMap ou Gráfico de Colunas) você acha mais apropriada? Todos os participantes responderam que a técnica BrazilMap é a melhor para interpretar dados referentes aos estados do Brasil.

$\mathrm{Na}$ etapa 1, os participantes analisaram as técnicas BrazilMap (Figura 4) e Gráfico de Colunas (Figura 5) com os dados dos programas Bolsa Alimentação e Bolsa Escola no ano de 2005. Nesta etapa, a pergunta foi: qual estado teve a maior diferença no número de pessoas atendidas nos dois programas apresentados (Bolsa Escola e Bolsa Alimentação)?

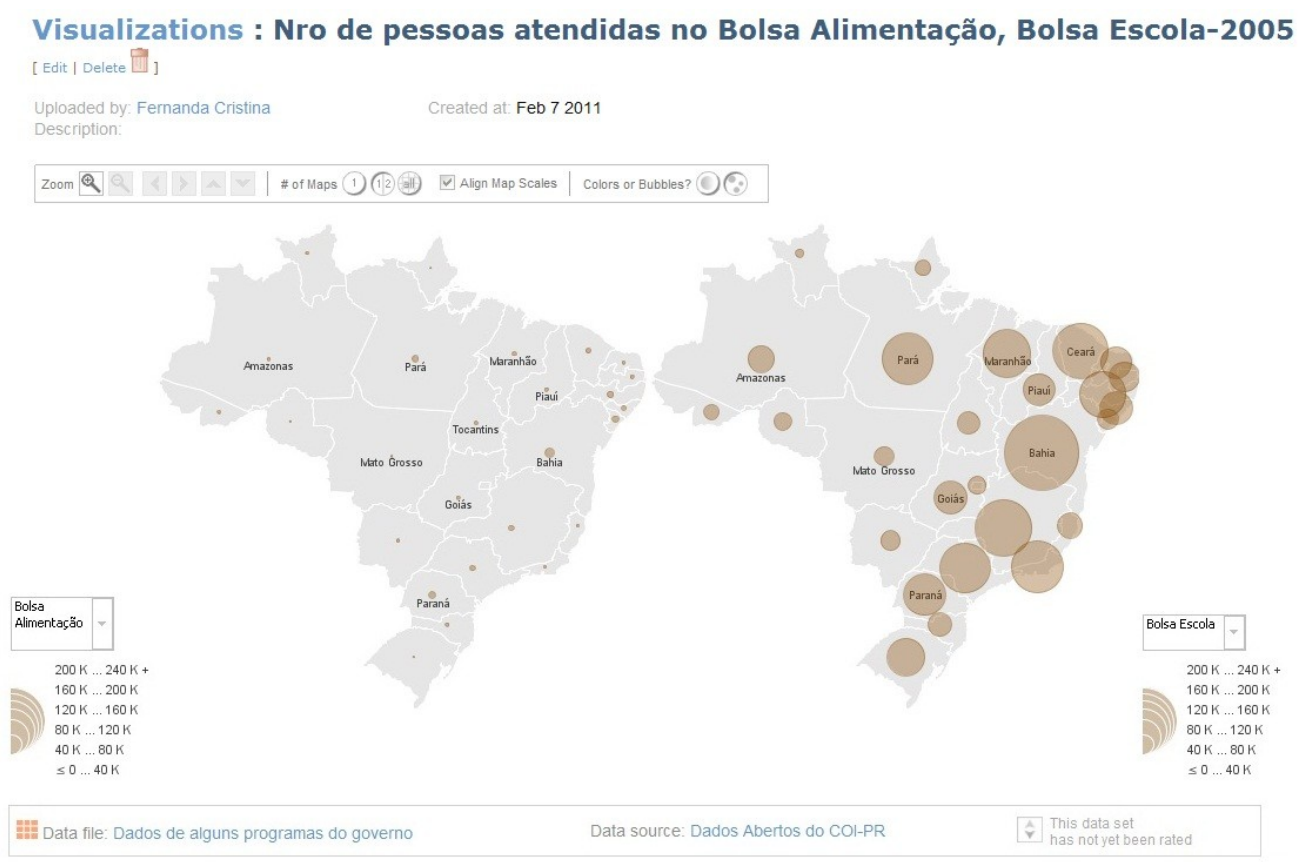

Figura 4. Técnica BrazilMap - Etapa 1

Todos responderam corretamente a questão. Duas pessoas comentaram que a técnica BrazilMap é melhor para visualizar as informações. No entanto, conforme descrito no gráfico da Figura 6a, o tempo gasto para responder a pergunta usando o Gráfico de Colunas foi, significativamente, menor para todos os participantes (Figura 6a). 


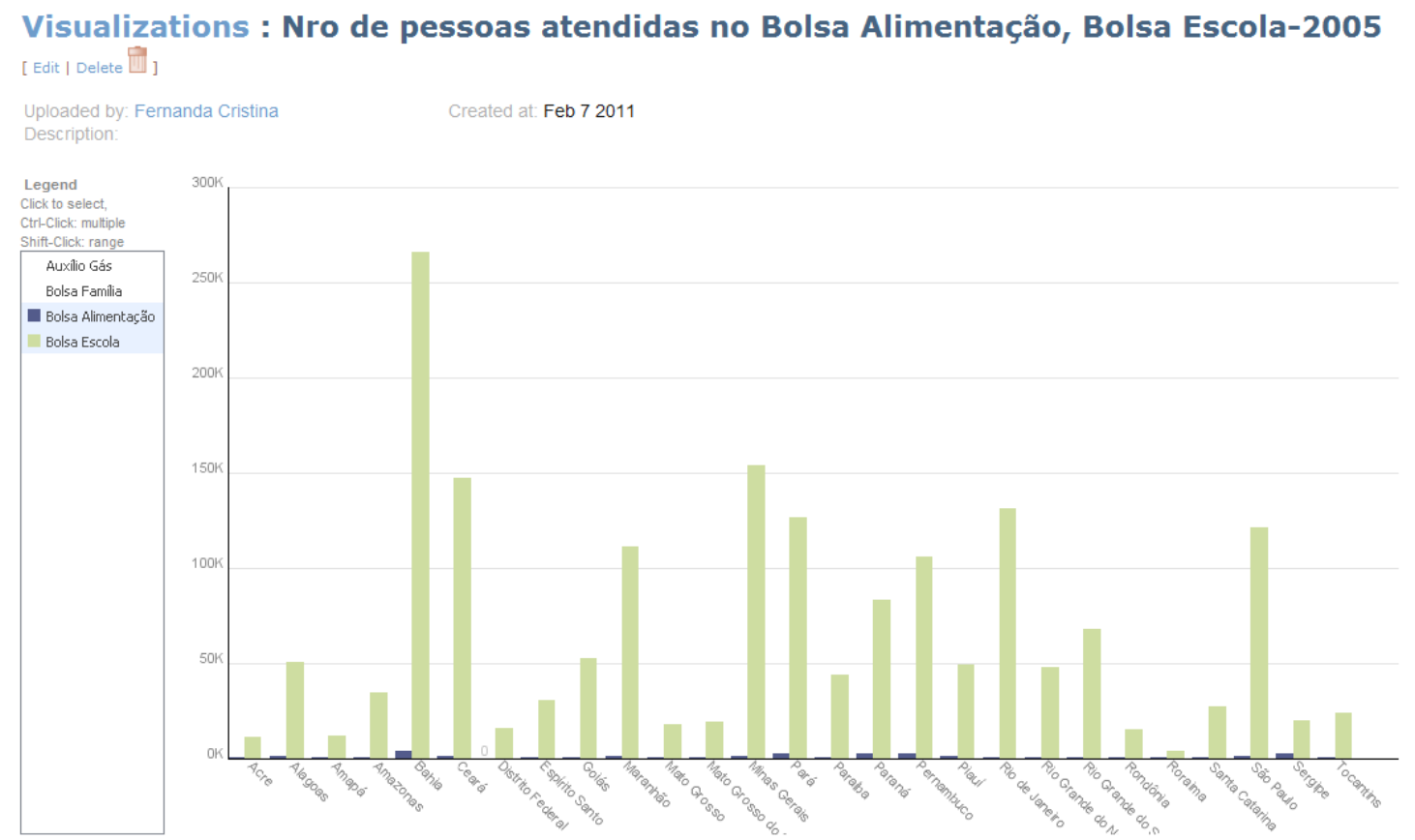

Figura 5. Técnica Gráfico de Colunas - Etapa 1

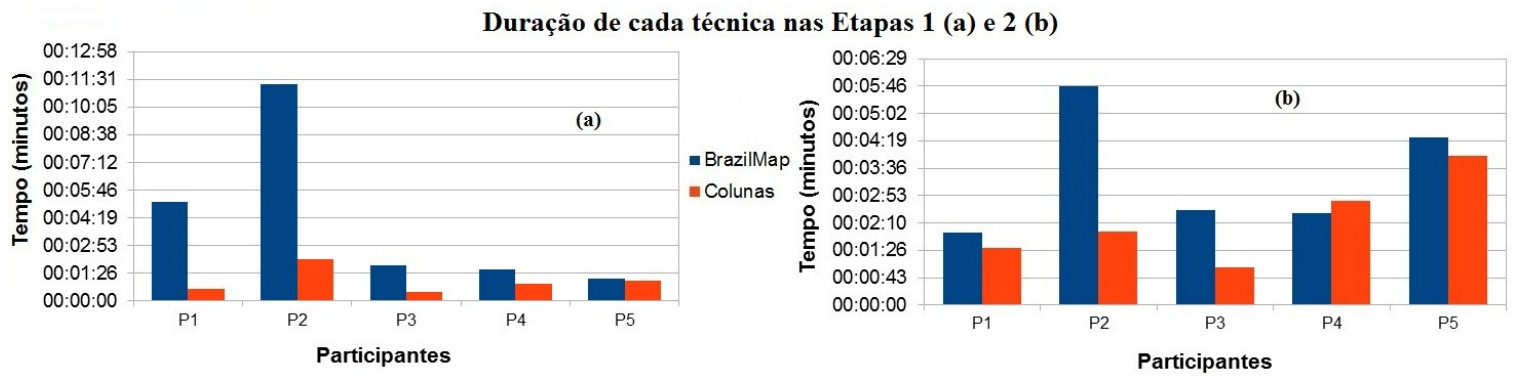

Figura 6. Tempo de resposta nas Etapas 1 (a) e 2 (b)

$\mathrm{Na}$ etapa 2, as técnicas BrazilMap (Figura 7) e Gráfico de Colunas (Figura 8) foram geradas com o número de famílias atendidas pelo programa Bolsa Família no ano de 2005. Após analisar as técnicas, os participantes tinham que realizar a seguinte tarefa: escreva o nome do estado com maior número de pessoas atendidas pelo programa Bolsa Família em cada região.

Nessa etapa, $80 \%$ dos participantes iniciaram a realização da tarefa pela técnica BrazilMap. No entanto, a tarefa foi realizada com mais rapidez utilizando o Gráfico de Colunas (Figura 6b). 




Figura 7. Técnica BrazilMap - Etapa 2

Visualizations: Número de pessoas atendidas pelo programa Bolsa Família (2005)

Uploaded by. Fernanda Cristina Created at. Feb 72011


Figura 8. Técnica Gráfico de Colunas - Etapa 2

Ao final de cada etapa, os participantes escreveram suas observações a respeito das técnicas apresentadas. Ao analisar as observações, foi possível identificar três aspectos que foram comentados por todos os participantes: a comparação entre o conhecimento adquirido em cada visualização, a facilidade e a dificuldade de uso das técnicas em cada etapa (Figuras 9a e 9b):

- Conhecimento adquirido: os participantes destacaram o conhecimento adicional a partir de cada técnica. Por exemplo, um participante registrou (analisando a técnica 
BrazilMap na etapa 1) que os estados da região Norte como Roraima, Amapá e Acre apresentaram o menor número de famílias atendidas nos programas Bolsa Alimentação e Bolsa Escola, em comparação com os outros estados. Como pode ser visto na figura 9a e 9b, na etapa 1, o número de observações quanto ao conhecimento adquirido foi igual nas duas visualizações. Contudo, na etapa 2, os participantes relataram um maior número de novos conhecimentos com o gráfico de colunas.

- Difícil visualização: os participantes relataram as características das técnicas que dificultaram a leitura e interpretação das informações. Por exemplo, um participante relatou que encontrou dificuldade para identificar os estados por região no Gráfico de Colunas durante a execução da tarefa da etapa 2. Outro participante citou que as cores com tonalidades semelhantes (na técnica BrazilMap nas etapas 1 e 2) e as bolhas (na técnica BrazilMap na etapa 1) dificultaram a análise e interpretação dos dados.

- Fácil visualização: os participantes relataram as características das técnicas que facilitaram a análise das informações. Por exemplo, dois participantes comentaram que para realizar a tarefa da etapa 2, a técnica BrazilMap não oferece dificuldades na análise e interpretação dos dados.



Figura 9. Número de Observações nas Etapas 1 (a) e 2 (b)

As observações dos participantes quanto aos aspectos: conhecimento adquirido, difícil e fácil visualização, estão relacionados com o conceito de transparência de dados públicos. Pois, segundo Silva (2010), a transparência dos dados governamentais permite que o cidadão tome consciência do fluxo de informações presentes nos setores públicos. Sendo assim, ao utilizar técnicas de InfoVis para visualizar os dados disponibilizados, o cidadão, além de acessar os dados, pode compreender melhor a informação transmitida.

\section{Considerações Finais}

De acordo com a pergunta feita na introdução do experimento, esperava-se que as interações com as visualizações geradas pela técnica BrazilMap tivessem melhores resultados, pois todos responderam que a representação visual BrazilMap era a melhor para analisar dados relacionados aos estados brasileiros.

Contudo, os resultados da etapa 1 sugerem que a técnica Gráfico de Colunas é a mais indicada para a análise de discrepância de dados. Uma justificativa para este resultado é que as pessoas têm mais acesso para manipular e analisar dados originados a partir desta técnica do que a BrazilMap. Esta constatação pode ser confirmada a partir dos dados representados nas figuras 6a e 9a. A maioria dos participantes gastou menos 
de um minuto (Figura 6a) para responder a pergunta. Além disso, de acordo com a Figura 9a, segundo as observações dos participantes, a técnica Gráfico de Colunas apresenta características que permitem uma visualização mais fácil.

Na segunda etapa, para realizarem a tarefa, os participantes levaram mais tempo na técnica BrazilMap (Figura 6b). Mas é importante ressaltar que, enquanto os participantes observavam a técnica Gráfico de Colunas para completar a tarefa, muitos retornavam à técnica BrazilMap para confirmar a região dos estados. Além disso, no quesito "Fácil Visualização" (Figura 9b), essa técnica foi considerada melhor que o Gráfico de Colunas.

Deste modo, foi possível observar diferenças significativas quanto à adequação de cada uma das técnicas. Para os dados analisados, por se tratar de informações a respeito de localidades geográficas (estados e regiões), a primeira impressão induz a escolha da técnica BrazilMap para divulgação das informações. Contudo, os resultados mostram que é necessário investigar cuidadosamente qual o tipo de conhecimento que se pretende criar.

Para dar continuidade ao trabalho, outros tipos de dados e visualizações serão investigados, incluindo, a divulgação de dados textuais. O objetivo é ampliar o escopo desta análise, tanto em relação ao público alvo quanto as técnicas utilizadas para que seja possível definir um conjunto de regras que possam facilitar a escolha destas técnicas pelos diversos órgãos públicos.

\section{Referências Bibliográficas}

Brazil Map, 2011. Disponível em: <http://www958.ibm.com/software/data/cognos/manyeyes/page/World_Map.html >. Acessado em 31 de jan. 2011.

Bubble Chart, 2011. Disponível em: <http:/www958.ibm.com/software/data/cognos/manyeyes/page/Bubble_Chart.html>. Acessado em 31 de jan. 2011.

Canárias, 2011. Portal do Governo das Canárias. Disponível em: $<$ http://visor.grafcan.es/visorweb $>$. Acessado em 16 de fev. 2011.

Card, S. K, Mackinlay, J. D. (1997) "The Structure of the Information Visualization Design Space". In: IEEE SYMPOSIUM ON INFORMATION VISUALIZATION, INFOVIS. Proceedings...[S.1.]:IEEE, p. 92-99.

Card, S. K, Mackinlay, J. D., Shneiderman, B. (1999) "Readings in Information Visualization: Using Vision to Think”. Morgan Kaufman Publishers, 1999.

COI, 2011. Disponível em: <https://i3gov.planejamento.gov.br/coi/>. Acessado em 24 de jan. 2011.

FGI, 2011. Free Government Information. Disponível em: <http://freegovinfo.info/>. Acessado em 16 de fev. 2011.

Lengler R., Eppler M. (2007) "Towards A Periodic Table of Visualization Methods for Management". IASTED Proceedings of the Conference on Graphics and Visualization in Engineering (GVE 2007), Clearwater, Florida, USA. 
Many 2011. Eyes, Disponível em: <http://www958.ibm.com/software/data/cognos/manyeyes/>. Acessado em 24 de jan. 2011.

Nascimento, Hugo A. D. do e Ferreira, Cristiane B. R. (2005) "Visualização de Informações - uma abordagem prática". In: A Universalidade da Computação: Um Agente de Inovação e Conhecimento. 2005, São Leopoldo - RS. Anais do XXV Congresso da Sociedade Brasileira de Computação. P. 1262 - 1312.

Projeto Many Eyes, 2011. Disponível em: $<$ http://fernandaviegas.com/democratizing_viz.html>. Acessado em 14 de fev. 2011.

Relatórios 2011. PAC, Disponível em: $<$ http://www.brasil.gov.br/pac/relatorios/nacionais/11o-balanco-4-anos $>$. Acessado em 24 de jan. 2011.

Ribeiro, D. M. (2009) "Visualização de Dados na Internet”. Dissertação de Mestrado em Tecnologias da Inteligência e Design Digital. PUC-SP, São Paulo.

ScreenCam, 2011. Disponível em: $<$ http://www.baixaki.com.br/download/autoscreenrecorder.htm $>$. Acessado em 31 de jan. 2011.

Shneiderman, B. (1992) "Tree Visualization with TreeMaps: 2D Space-Filling Approach". ACM Transactions on Graphics, v. 11, n. 1(January), p. 92-99.

Silva, Celmar G. da (2007) "Considerações sobre o uso de Visualização de Informação no auxílio à gestão de informação". In: XXXIV Seminário Integrado de Software e Hardware (SEMISH). 2007, Rio de Janeiro - RJ. Anais do XXVII Congresso da SBC. P. $2070-2084$.

Silva, Daniela B. da (2010) "Transparência na Esfera Pública Interconectada". Dissertação de Mestrado em Processos Midiáticos: Tecnologia e Mercado. Faculdade Cásper Líbero, São Paulo.

Souza, D. K. (2008) "Utilização de Técnicas de Visualização para a Recomendação de Substitutos". Dissertação de Mestrado. Programa de Engenharia de Sistemas e Computação. COPPE/UFRJ, Rio de Janeiro.

Tag Cloud, 2011. Disponível em: <http://www958.ibm.com/software/data/cognos/manyeyes/page/Tag_Cloud.html $>$. Acessado em 31 de jan. 2011.

Visual Literacy, 2010. Disponível em: $<$ http://www.visualliteracy.org/periodic_table/periodic_table.html>. Acessado em 16 de dez 2010.

Visualizações Many Eyes, 2011. Disponível em: <http://www958.ibm.com/software/data/cognos/manyeyes/users/Fernanda+Cristina/visualizations >. Acessado em 14 de fev. 2011.

Word 2011. Tree, Disponível em: <http://www958.ibm.com/software/data/cognos/manyeyes/page/Word_Tree.html $>$. Acessado em 31 de jan. 2011. 